TITLE:

\title{
Theory of solvation-induced reentrant phase separation in polymer solutions
}

\author{
AUTHOR(S):
}

Matsuyama, Akihiko; Tanaka, Fumihiko

\section{CITATION:}

Matsuyama, Akihiko ...[et al]. Theory of solvation-induced reentrant phase separation in polymer solutions. Physical Review Letters 1990, 65(3): 341-344

\section{ISSUE DATE:}

1990-07-16

URL:

http://hdl.handle.net/2433/49180

RIGHT:

Copyright 1990 American Physical Society 


\title{
Theory of Solvation-Induced Reentrant Phase Separation in Polymer Solutions
}

\author{
Akihiko Matsuyama \\ Department of Applied Physics, Faculty of Engineering, University of Tokyo, Bunkyo-ku, Tokyo I13, Japan \\ Fumihiko Tanaka \\ Department of Physics, Faculty of General Education, Tokyo University of Agriculture and Technology, \\ Fuchu-shi, Tokyo 183, Japan \\ (Received 20 December 1989)
}

\begin{abstract}
A simple model of solvation is introduced to describe lower critical solution points in polymer solutions. Our theory predicts that physical bond formation between polymer segments and solvents can be a major cause of miscibility gaps showing a closed-loop or hourglass shape. Relative positions of upper and lower critical solution points are examined for the various molecular weights of the polymer. The result is compared with the observed phase diagram of polyethylene glycol in water.
\end{abstract}

PACS numbers: $64.70 .-\mathrm{p}, 64.75 .+\mathrm{g}, 81.30 . \mathrm{Dz}$

In certain binary mixtures, lower critical solution points (LCST) are often observed. Typical examples are nicotine in water, ${ }^{\prime}$ polyethylene glycol in water, ${ }^{2-4}$ and ethylene-vinyl acetate (EVA) blends with chlorinated polyethylene (CPE). ${ }^{5}$ There is evidence that these solutions contain a complex formed by molecules of different species, and that the formation of the complex is caused by a hydrogen bond. ${ }^{5-9}$ The microscopical explanations of these properties in small molecules have been reviewed by Walker and Vause. ${ }^{9}$ Recent experimental and theoretical studies ${ }^{7}$ have also suggested that an interaction of the hydrogen-bonding type prevails in EVA-CPE blends.

In this paper we examine a possible mechanism for the appearance of LCST in polymer solutions from a molecular point of view. The current understanding of the phase behavior in polymer solutions is based on the cooccurrence of solvation (hydration) and phase separation on the temperature-concentration plane. Our theory predicts that a physical bond formation between polymer segments and solvents causes peculiar types of phase separation, such as a closed-loop shape and an hourglass shape. The result is compared with the observed phase diagram for polyethylene glycol in water. ${ }^{4}$

When molecules form hydrogen bonds, the solution contains polydisperse molecular aggregates. It has been reported that the polydispersity is an important factor in associating liquids, ${ }^{6}$ including micellar solutions ${ }^{10}$ and physical gels. ${ }^{11}$ The theoretical understanding of these solutions must incorporate the size distributions of such aggregates, strongly dependent on concentration and temperature. We consider here the polydisperse polymer-solvent complexes formed by hydrogen bonds.

Consider a binary mixture of polymer and solvent. Each polymer chain is assumed to carry $f$ identical functional groups which do not interact with each other but are capable of forming physical bonds with the solvent molecules by pairwise association. The bonding energy considered here is on the order of the thermal energy, so that bonding-unbonding equilibrium is easily established by thermal activation. In thermal equilibrium, each polymer chain is associated with a certain number of solvent molecules. If $m$ groups among $f$ on a chain are bonded with the solvents, we call them $m$ cluster in the following. We include a pure chain in the case of $m=0$. To derive the population of such clusters, we now consider the thermodynamics of the system.

The free energy of our system can be constructed by the sum of the two terms, $F=F_{\text {rea }}+\Delta F_{\text {mix }}$, each required in two different steps starting from the reference state where pure solvent and pure polymer molecules are prepared separately. The first term $F_{\text {rea }}$ is the free energy of the quasireference state where the clusters and the unbonded free solvents are prepared separately. It is written as $F_{\text {rea }}=N_{0} \mu_{0}^{0}+\sum N_{m+1} \mu_{m+1}^{0}$ in terms of the chemical potential $\mu_{m+1}^{0}$ of a single isolated $m$ cluster, where $N_{m+1}$ is the number of $m$ clusters and $N_{0}$ the unbonded free solvent molecules. The second term $\Delta F_{\text {mix }}$ describes the free-energy change required in the process of mixing the thus constructed clusters with the free solvents. According to the lattice theory of Flory-Huggins, ${ }^{12}$ the free energy $\Delta F_{\mathrm{mix}}$ is given by

$\beta \Delta F_{\mathrm{m} \mathrm{x}}=N_{t}\left(\phi_{0} \ln \phi_{0}+\sum_{m=0}^{f} \frac{\phi_{m+1}}{n+m} \ln \phi_{m+1}+\chi \phi(1-\phi)\right)$,

where $\phi_{0}$ is the volume fraction of the free solvent molecules, $\phi_{1}$ is that of the unbonded polymer, $\phi_{m+1}$ $(m=1,2, \ldots, f)$ is that of the $m$ clusters, $\beta \equiv 1 / k_{B} T, N_{t}$ is the total number of lattice cells in the system, $n$ is the number of segments on a polymer, and $\chi$ is the solventsolute interaction parameter. The total volume fraction $\phi$ of the polymer is given by $\phi=\Sigma[n /(n+m)] \phi_{m+1}$. For binary systems, we have $\phi_{0}+\sum \phi_{m+1}=1$ by definition.

In thermal equilibrium, each molecule is in chemical equilibrium through bonding and unbonding processes. The multiple chemical equilibria conditions ${ }^{6}$ are given by

$$
\mu_{m+1}=\mu_{1}+m \mu_{0} \quad(m=0,1,2, \ldots, f),
$$


where $\mu_{0}=\partial F / \partial N_{0}$ is the chemical potential of the free solvent, $\mu_{m+1}=\partial F / \partial N_{m+1}$ is that of the $m$ cluster, and $\mu_{1}=\partial F / \partial N_{1}$ is that of the unbonded polymer. Explicitly we have

$$
\begin{aligned}
& \beta \mu_{0}=\beta \mu_{0}^{0}+\ln \phi_{0}+1-\left(\phi_{0}+\frac{\phi}{n}\right)+\chi \phi^{2}, \\
& \beta \mu_{m+1}=\beta \mu_{m+1}^{0}+\ln \phi_{m+1}+1-(n+m)\left(\phi_{0}+\frac{\phi}{n}\right) \\
& +n \chi\left[(1-\phi)^{2}+\frac{m}{n} \phi^{2}\right] .
\end{aligned}
$$

Substituting Eq. (3) into Eq. (2) we have $\phi_{m+1}$ $=K_{m} \phi_{1} \phi_{0}^{m}$ for the volume fraction of $m$ clusters, where the association constant $K_{m}$ is expressed as $K_{m}$ $=\exp \left(m-\Delta_{m}\right)$ in terms of the free-energy difference $\Delta_{m}$, which is defined by $\Delta_{m} \equiv \beta\left(\mu_{m+1}^{0}-\mu_{1}^{0}-m \mu_{0}^{0}\right)$. Splitting the free-energy change $\Delta_{m}$ into the entropy $\Delta S$ and the enthalpy $\Delta H$ terms, we have $\Delta_{m}=\beta(\Delta H-T \Delta S)$. The entropy change $\Delta S$ consists of two parts: $\Delta S=\Delta S_{\text {com }}+\Delta S_{\text {dis. }}$. The combinatorial entropy $\Delta S_{\text {com }}$ is given by $\Delta S_{\text {com }}=k_{B} \ln W_{m}$, where $W_{m}$ is the number of ways to select $m$ functional groups out of $f$ on a chain, and is given by the binomial coefficient. We then have

$$
\Delta S_{\mathrm{com}}=k_{B} \ln \left(\frac{f !}{m !(f-m) !}\right) .
$$

The configurational entropy change $\Delta S_{\text {dis }}$ for an $m$ cluster formation is given by $\Delta S_{\mathrm{d} \text { s }}=S(n, m)-S(n, 0)$ - $m S(1,0)$, where $S(n, m)$ is the configurational entropy of a single $m$ cluster whose segment number is $n+m$. By the use of "entropy of disorientation," $S(n, m)$ is given by

$$
S(n, m)=k_{B} \ln \left(\frac{(n+m)_{z}(z-1)^{n+m-2}}{\sigma \exp (n+m-1)} w^{m}\right),
$$

where $z$ is the coordination number of a quasilattice, $\sigma$ is the symmetry number of the cluster, and $w$ is a constant giving the statistical weight whose logarithm is the local entropy change for a bond formation. Using Eq. (5), $\Delta S_{\text {dis }}$ is expressed as

$$
\Delta S_{\mathrm{dis}}=k_{B} \ln \left(\frac{n+m}{n} \lambda_{0}^{m}\right),
$$

where $\lambda_{0} \equiv \sigma(z-1)^{2} w / z e$ is a parameter related to the local entropy change ${ }^{9}$ for a single bond formation. The energy change $\Delta H$ is given by $\Delta H=-m \Delta \varepsilon$, where $\Delta \varepsilon$ $(>0)$ is the energy required for a bond formation. Substituting the above results into $\Delta_{m}$, we find the association constant $K_{m}$ is given by

$$
K_{m}=\frac{n+m}{n}\left(\frac{f !}{m !(f-m) !}\right) \lambda(T)^{m},
$$

where $\lambda(T)$ is defined by $\lambda(T) \equiv \lambda_{0} \exp (\beta \Delta \varepsilon)$.
By the normalization condition together with multiple equilibria, the volume fraction $\phi_{0}$ of the free solvents is given as a function of $\phi$ and $\lambda(T)$ :

$$
\phi_{0}=[\lambda-1-(1+P) \lambda \phi+\sqrt{D(\phi)}] / 2 \lambda,
$$

where $P \equiv f / n$ and

$$
D(\phi) \equiv[\lambda-1-(1+P) \lambda \phi]^{2}+4 \lambda(1-\phi) .
$$

When $P=0$, we have $\phi_{0}=1-\phi$.

In the following we consider the equilibrium solution properties. It is convenient to consider the free energy per lattice cell: $f_{u} \equiv F / N_{t}$.

The spinodal line is obtained ${ }^{12}$ from $\partial^{2} f_{u} / \partial \phi^{2}=0$, or equivalently from $\partial \mu_{0} / \partial \phi=0$. This leads to

$$
\frac{1}{n \phi}+\frac{\phi_{0}-1}{\phi_{0} \phi}\left(\frac{d \phi_{0}}{d \phi}\right)-2 \chi=0 \text {. }
$$

The condition for the two phases to be in thermal equilibrium is given by $\mu_{0}\left(\phi^{\prime}\right)=\mu_{0}\left(\phi^{\prime \prime}\right)$ and $\mu_{m+1}\left(\phi^{\prime}\right)$ $=\mu_{m+1}\left(\phi^{\prime \prime}\right)$ for $m=0,1,2, \ldots, f$. Because of the multiple equilibria conditions $\mu_{m+1}=\mu_{1}+m \mu_{0}$, however, the coexistence curves (binodals) of the phase equilibrium are derived by the coupled equations $\mu_{0}\left(\phi^{\prime}\right)=\mu_{0}\left(\phi^{\prime \prime}\right)$ and $\mu_{1}\left(\phi^{\prime}\right)=\mu_{1}\left(\phi^{\prime \prime}\right)$, where $\phi^{\prime}$ and $\phi^{\prime \prime}$ are the polymer concentrations in the higher and the lower concentration phases, respectively.

The osmotic pressure $\pi$ is related to the solvent's chemical potential by $\pi \beta a^{3}=-\beta \Delta \mu_{0}$, where $\Delta \mu_{0} \equiv \mu_{0}$ $-\mu_{0}^{0}$. In the dilute regime we can expand $\phi_{0}$ in powers of the concentration $\phi$. Substitution of it into $\Delta \mu_{0}$ yields the dimensionless second virial coefficient in a simple form:

$$
A_{2}=\frac{1}{2}-\{\chi-\eta(1+\eta / 2)\},
$$

where $\eta \equiv P \lambda /(\lambda+1)$. The parameter $\chi$ is always positive and increases with temperature decrease. ${ }^{12}$ As $\lambda(T)$ is always positive, the effect of solvation reduces the net interaction between the two components. The number-

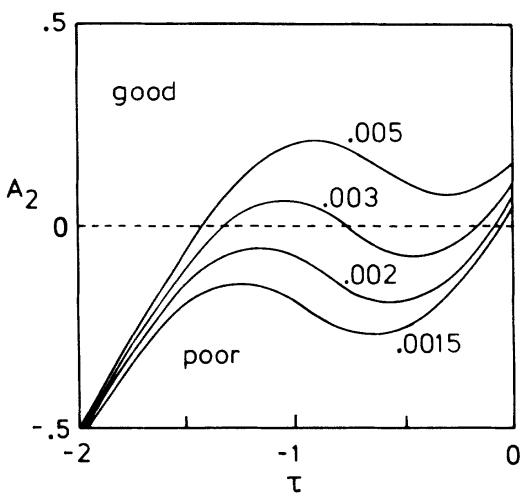

FIG. 1. Second virial coefficient $A_{2}$ against the reduced temperature $\tau$. The entropy parameter $\lambda_{0}$ is varied from curve to curve. 
average cluster size $\langle m\rangle$, which also equals the average number of solvent molecules bonded on the polymer, is given by $\langle m\rangle=\sum m N_{m+1} / \sum N_{m+1}$. Hence we have $\langle m\rangle$ $=f x /(1+x)$, where $x$ is defined as $x \equiv \lambda \phi_{0}$.

For the numerical calculation we introduce the reduced temperature $\tau$ by the definition $\tau \equiv 1-\Theta_{0} / T$, where $\Theta_{0}$ is the unperturbed theta temperature which satisfies an equation $\chi\left(\Theta_{0}\right)=\frac{1}{2}$. In terms of $\tau$, the $\chi$ pa-
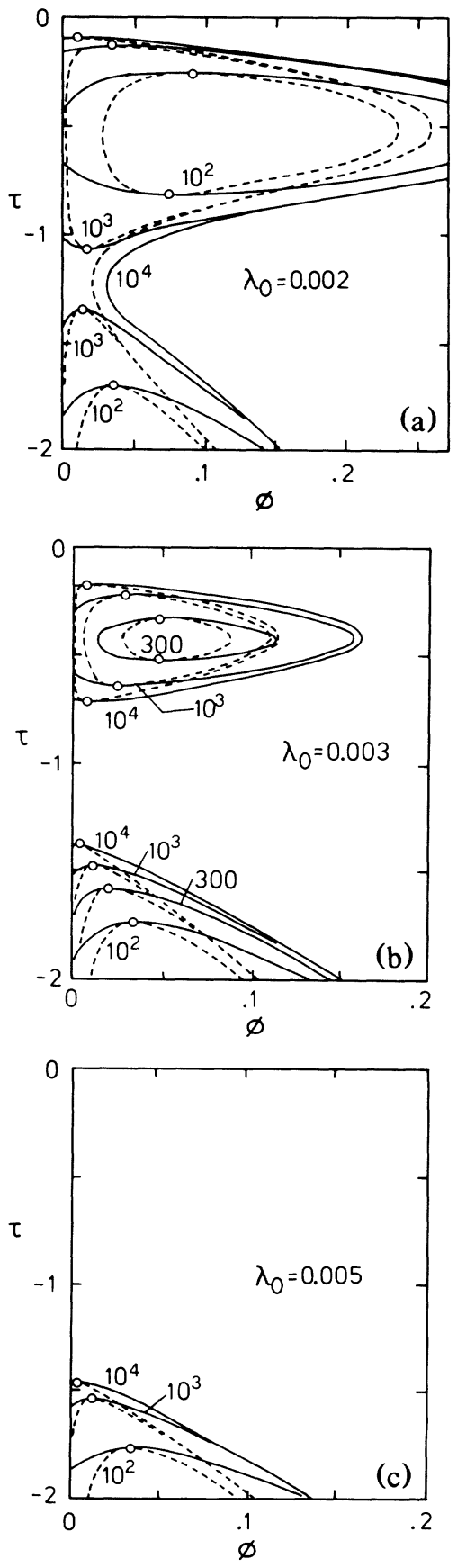

FIG. 2. Phase diagrams on the temperature-concentration plane. The solid (dashed) curves correspond to the binodal (spinodal) curves. See text for details. rameter and $\lambda(\tau)$ can be expressed as $\chi=\frac{1}{2}-\psi_{1} \tau$ and $\lambda(\tau)=\lambda_{0} \exp [\gamma(1-\tau)]$, respectively, where $\gamma \equiv \Delta \varepsilon / k_{B} \Theta_{0}$. We then have five parameters characterizing the system: functionality $f$ of a polymer, number $n$ of the statistical units on a polymer chain, entropy parameter $\lambda_{0}$ for a single bond formation, dimensionless bonding energy $\gamma$, and unperturbed polymer-solvent interaction $\psi_{1}$.

Figure 1 shows the second virial coefficient $A_{2}$ plotted against the reduced temperature $\tau$. We have fixed $P=1$, $\psi_{1}=1$, and $\gamma=3.5$ for a typical example. The entropy parameter $\lambda_{0}$ is varied from curve to curve. The temperature for which $A_{2}=0$ is the $\Theta$ temperature. The region where $A_{2}<0$ corresponds to a poor solvent condition and the region where $A_{2}>0$ to a good solvent condition. The second virial coefficient exhibits a maximum and a minimum as a function of the temperature. Since the curve for $\lambda_{0}=0.003$ has three $\Theta$ temperatures, a closedloop coexistence curve appears in the poor solvent region at the higher-temperature side, as shown in Fig. 2(b).

Figure 2 shows the phase diagrams on the temperature-concentration plane. The entropy parameter $\lambda_{0}$ is changed from Fig. 2(a) to Fig. 2(c). The segment number $n$ is varied from curve to curve. The critical solution points are indicated by the open circles. The solid (dashed) curves correspond to the binodal (spinodal) curves. The temperature of the maximum of each coexistence curve corresponds to the upper critical solution temperature (UCST) and the temperature of it is minimum to the lower critical solution temperature (LCST). As shown in Fig. 2(a) we have, for lower molecular weights, closed-loop coexistence curves (CLC) showing a UCST and a LCST, and the lower coexistence curves showing a UCST. For larger values of the segment number $n$, we have an hourglass shape of the phase diagram such as observed for polyethylene glycol in $t$ butyl acetate. ${ }^{13}$ Figure 2(b) shows the phase diagram for $\lambda_{0}=0.003$. As the segment number $n$ increases, the



FIG. 3. Comparison of the theoretical calculation with the observed phase diagram for polyethylene glycol (PEO) in water. 


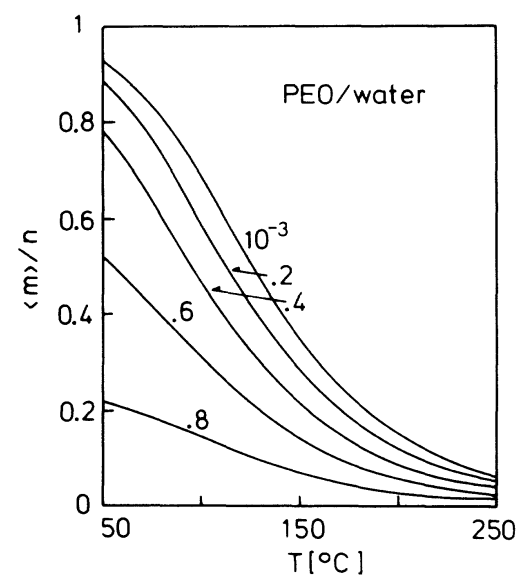

FIG. 4. Average number $\langle m\rangle$ of the solvent molecules (water) bonded to a polymer chain (PEO) shown against the temperature.

LCST of the CLC is lowered, while the UCST of the CLC and of the lower coexistence curves are raised. The hourglass phase diagram, however, does not appear since we have three $\Theta$ temperatures. The CLC is absent for $n=100$. As the entropy parameter $\lambda_{0}$ increases further, the CLC disappears as shown in Fig. 2(c), and we have the coexistence curves with the UCST only. These phase diagrams appear as the results of the co-occurrence of solvation and phase separation.

Figure 3 shows the comparison of the theoretical calculation with the observed phase diagram for polyethylene glycol (PEO) in water. ${ }^{4}$ The number-average molecular weights of the PEO were estimated ${ }^{4}$ to be 2170-10×105: open circles, 2180; solid circles, 2270; open triangles, 2290; solid triangles, 8000 ; vertical halffilled circles, $14.4 \times 10^{3}$; horizontal half-filled circles, $21.2 \times 10^{3}$; open squares, $1020 \times 10^{3}$. The solid curves show the calculated binodals. The segment number $n$ in our numerical calculation is varied from curve to curve. The following values of the parameters are used to fit the experimental data: $\psi_{1}=1, \Theta_{0}=730 \mathrm{~K}, \gamma=6, P=1$, and $\lambda_{0}=1.66 \times 10^{-5}$. The fit of theoretical curves to the experimental data is very good.

Figure 4 shows the average solvation number $\langle m\rangle$ versus the temperature. The volume fraction of PEO is varied from curve to curve. Near the LCST, the value of $\langle m\rangle$ rapidly decreases with increasing temperature. These analyses demonstrate that the hydrogen bond for- mation between the ether oxygen molecules of PEO and water molecules causes the LCST. When temperature is increased, the hydrogen bonds break and water becomes a poorer solvent for the polymer. ${ }^{6}$

In conclusion, a simple theory presented here can describe the lower critical solution point as the results of the co-occurrence of solvation and phase separation. The mixing entropy produced by solvation is the main cause of the LCST. The concept of solvation (hydration) is of central importance in other aqueous polymer solutions, and also in nonionic surfactants ${ }^{2}$ and nonionic gels ${ }^{14}$ in water.

'C. S. Hudson, Z. Phys. Chem. 47, 113 (1904).

2J. C. Lang and R. D. Morgan, J. Chem. Phys. 73, 5849 (1980); M. Corti, C. Minero, and V. Degiorgio, J. Phys. Chem. 88, 309 (1984); M. Kahlweit, E. Lessner, and R. Strey, J. Phys. Chem. 87, 5032 (1983); 88, 1937 (1984).

${ }^{3}$ G. N. Malcolm and J. S. Rowlinson, Trans. Faraday Soc. 53, 921 (1957).

${ }^{4}$ S. Saeki, N. Kuwahara, M. Nakata, and M. Kaneko, Polymer 17, 685 (1976).

${ }^{5}$ D. J. Walsh, J. S. Higgins, and S. Rostami, Macromolecules 16, 388 (1983).

${ }^{6} \mathrm{~V}$. Degiorgio, in Physics of Amphiphiles: Micelles, Vesicles, and Microemulsion, edited by V. Degiorgio and M. Corti (North-Holland, Amsterdam, 1985); R. Kjellander and E. Florin, J. Chem. Soc. Faraday Trans. 77, 2053 (1981).

${ }^{7}$ M. M. Coleman, E. J. Moskala, P. C. Painter, D. J. Walsh, and S. Rostami, Polymer 24, 1410 (1983); P. C. Painter, Y. Park, and M. M. Coleman, Macromolecules 22, 570 (1989); 22, 580 (1989); 22, 586 (1989).

${ }^{8}$ G. R. Anderson and J. C. Wheeler, J. Chem. Phys. 69, 3403 (1978); 73, 5778 (1980); J. S. Walker and C. A. Vause, Phys. Lett. 79A, 421 (1980); R. E. Goldstein and J. S. Walker, J. Chem. Phys. 78, 1492 (1983).

${ }^{9}$ J. S. Walker and C. A. Vause, Sci. Am. 256, No. 5, 90 (1987).

${ }^{10}$ D. Blankschtein, G. M. Thurston, and G. B. Benedek, Phys. Rev. Lett. 54, 955 (1985); J. Chem. Phys. 85, 7268 (1986).

I'F. Tanaka, Macromolecules 22, 1988 (1989); F. Tanaka and A. Matsuyama, Phys. Rev. Lett. 62, 2759 (1989).

${ }^{12}$ P. J. Flory, Principles of Polymer Chemistry (Cornell Univ. Press, Ithaca, 1953), Chaps. 12 and 13.

${ }^{13}$ S. Saeki, N. Kuwahara, S. Konno, and M. Kaneko, Macromolecules 6, 247 (1973); 8, 799 (1975).

${ }^{14}$ Y. Hirokawa and T. Tanaka, J. Chem. Phys. 81, 6379 (1984). 